\title{
A Power Model for Intrusion Detection and Defense System in Ad Hoc Network
}

\author{
S.V. Sonekar \\ JDCOE,Nagpur
}

NIT Nagpur
NIT

\author{
N.A. Mohota \\ JDCOE,Nagpur
}

\author{
V.D. Rughwani \\ VDCOE, Mouda
}

\begin{abstract}
One of the most important networking problems is assuring the network and its resources performing as expected. If network's behavior is unpredictable or unexpected, such a network is insecure. Therefore, to assure that a network performs as expected it must be secure. Many researchers in the field of network security have stated that network security is a process that tries to optimize two characteristics of secure systems.

Two characteristics are integrity and confidentiality. Having integrity means the system and its information remain unaltered by accidents or malicious attacks while confidentiality means the data that is in the system must be available only to users who are authorized to access and manipulate the information or resources on the network.

To assure that the network and its services are secure recommended practices call for system administrators to develop or implement a security system generally referred to as Intrusion Detection/Defense System (ID/DS).

In this paper the main area of discussion is the identification of malicious node and store unique id of the malicious node in the memory. This paper proposed a solution by combining few solutions and distilling the best from the solutions so that it can provide a better solution. Finally this paper concludes with implementing the solutions and the results obtained by the experiments using trading example. It is expected that after developing and implementing ID/DS the network will be more resistant to intrusion.
\end{abstract}

The full text of the article is not available in the cache. Kindly refer the IJCA digital library at www.ijcaonline.org for the complete article. In case, you face problems while downloading the full-text, please send a mail to editor at editor@ijcaonline.org 